\title{
Thermo-Optic Coefficient of Porous Silicon in the Infrared Region and Oxidation Process at Low Temperatures
}

\author{
David Martín-Sánchez, $\odot$ Miroslavna Kovylina, Salvador Ponce-Alcántara, \\ and Jaime García-Rupérez ${ }^{\mathrm{z}}$
}

Nanophotonics Technology Center (NTC), Universitat Politècnica de València, 46022 Valencia, Spain

\begin{abstract}
In this work, a porous silicon nanostructure has been fabricated by electrochemical means and used as a thermal sensor. The thermo-optic effect in the near infrared region has been experimentally studied based on spectroscopy measurements. Values of the thermo-optic coefficient between 3.2 and $7.9 \cdot 10^{-5} \mathrm{~K}^{-1}$ have been obtained, depending on the porosity, reaching a maximum thermal sensitivity of $91 \pm 3 \mathrm{pm} /{ }^{\circ} \mathrm{C}$ during the experiments carried out with the fabricated samples. Additionally, the oxidation process of the sensor at temperatures below $500 \mathrm{~K}$ has been studied, showing that the growth of the silicon oxide was dependent on the characteristics of the porous layers. Based on the experimental results, a mathematical model was developed to estimate the evolution of the oxidation process as a function of porosity and thickness.

(C) The Author(s) 2019. Published by ECS. This is an open access article distributed under the terms of the Creative Commons Attribution 4.0 License (CC BY, http://creativecommons.org/licenses/by/4.0/), which permits unrestricted reuse of the work in any medium, provided the original work is properly cited. [DOI: 10.1149/2.0341906jes]

(cc) BY
\end{abstract}

Manuscript submitted February 12, 2019; revised manuscript received March 14, 2019. Published March 23, 2019.

One of the most outstanding features of the silicon electrochemistry is the formation of pores by anodization. ${ }^{1}$ A fluoride-based electrolyte, typically containing hydrofluoric acid (HF), etches the silicon surface creating holes vertically, with diameters as small as $2 \mathrm{~nm} .^{2}$ This nanostructured material has been demonstrated to have an extremely high surface-to-volume ratio ${ }^{3}$ and a tunable refractive index (RI) with the porosity.

Porous silicon (PS) has attracted interest in many areas. In optoelectronics, the ability of controlling the optical properties and the emission of visible photoluminescence have enabled the development of optical devices operating in a wide range of the electromagnetic spectrum. ${ }^{4}$ Besides, the biocompatibility and biodegradability of PS along with the facility of surface functionalization have generated numerous applications in biomedicine for diagnosis and therapy. ${ }^{5}$ Furthermore, in the field of energy conversion, this material has been considered a potential substitute of bulk silicon in Li-ion batteries, enhancing the performance of the anodes due to its porous structure. ${ }^{6-8}$

The use of PS as a thermal transducer has proved its feasibility as an alternative to fiber-based temperature sensors and integrated photonic sensors. Hence, the study of thermal effects on PS is of fundamental importance, since many of its properties change with temperature and the stability and correct operation of the PS-based devices strongly depend on this feature. One way to evaluate the thermal effect on optical materials is by analyzing the thermo-optic effect (TOE). ${ }^{9}$ This phenomenon determines the variation of the RI with changes in temperature, which has been extensively exploited in optoelectronics for the development of a wide variety of devices, e.g. optical switches, tunable lasers or fiber-optic sensors. ${ }^{9}$ The thermo-optic coefficient (TOC) quantifies the TOE and is a generalized way of characterizing materials. Silicon has one of the highest TOC among thermo-optical materials, with $1.5 \cdot 10^{-4} \mathrm{~K}^{-1}$ in the near infrared region (NIR) ${ }^{11}$ and $5 \cdot 10^{-4} \mathrm{~K}^{-1}$ in the visible region. ${ }^{12}$ In consequence, PS also has a high TOC, but it is tunable with the porosity. In the visible range of the spectrum, some references report values between 0.3 and $1.0 \cdot 10^{-4} \mathrm{~K}^{-1}$, depending on the porosity. ${ }^{9,12}$ However, data concerning the TOC of PS in the NIR, to our knowledge, are missing. Only a mathematical estimation of $5.5 \cdot 10^{-5} \mathrm{~K}^{-1}$ for $1500 \mathrm{~nm}$ has been found in literature. ${ }^{9}$

Another result of the thermal effect on PS is the partial or total oxidation of the material, which permanently changes the physical structure. Oxidation of porous silicon can occur in a natural way, for example, when stored in ambient air at room temperature. The pore walls become partially oxidized by the so-called native oxide, changing the RI of the structure, the absorption coefficient and the luminescent properties. ${ }^{13}$ Otherwise, PS can be intentionally oxidized thermally in dry or wet environments in order to create a wider width

${ }^{\mathrm{z} E-m a i l: ~ j a i g a r r u @ n t c . u p v . e s ~}$ of the oxide layer. ${ }^{14}$ Thermal oxidation of PS occurs inside the pore walls as well as at the surface of the film. It is a rapid process that takes place mainly within a few minutes, depending on parameters such as the substrate composition, the porosity and the thickness. ${ }^{13}$ The degree of oxidation depends to a greater extent on the temperature, ${ }^{15}$ achieving a complete oxidation of the PS layer at around $1000 \mathrm{~K}$ in a dry environment. Lower temperatures partially oxidize the PS. According to Mawhinney et al., ${ }^{16}$ the first evidence of thermal oxidation is seen at $523 \mathrm{~K}$ using transmission infrared spectroscopy. The undesired oxidation of the nanostructure can impair its features, especially when the phenomenon occurs unexpectedly. However, there is a wide range of applications where oxidized PS is used. In optoelectronics, it can work as a protective coating and prevent aging of the optical devices. ${ }^{17}$ In biomedicine, the oxide layer maintains a stable and hydrophilic surface, which enhances the chemical modification with functional groups. Moreover, when applied to drug delivery, the oxide aids the dissolution of the PS particles. ${ }^{5}$

In our work, we fabricated electrochemically PS films in heavily doped p-type silicon wafers and characterized the thermal effect in the infrared at low temperatures. We performed spectroscopy measurements with the samples while varying the temperature from room temperature to $453 \mathrm{~K}$. The experimental thermal sensitivity was calculated as a function of the spectral shift divided by the temperature increase and the experimental TOC as a linear regression of the RI variation with temperature. In order to assess the obtained values, a mathematical model was used to calculate the theoretical sensitivity and TOC of each PS layer. We also analyzed the oxidation process of the nanostructure at temperatures below $500 \mathrm{~K}$, based on spectroscopy measurements. A dependency with the thickness and the porosity of the layer was found. As a result, we were able to develop a mathematical model that estimates the oxidation degree of the PS layer from a reflectance spectrum.

\section{Experimental}

PS was prepared by electrochemically etching p-type silicon wafers (boron doped, $<100>$ oriented, $0.01-0.02 \Omega \cdot \mathrm{cm}$ resistivity) purchased from MicroChemicals GmbH (Ulm, Germany). The electrolyte contained a mixture of Ethanol (EtOH), supplied by Scharlab S.L. (Barcelona, Spain), and hydrofluoric acid (HF) (48\% solution in water), purchased from BASF (Ludwigshafen am Rhein, Germany), in a 2:1 volumetric ratio. For cleaning purposes, all samples were pretreated during 5 minutes in a $3: 1$ volumetric mixture of sulfuric acid $\left(\mathrm{H}_{2} \mathrm{SO}_{4}\right)$ and hydrogen Peroxide $\left(\mathrm{H}_{2} \mathrm{O}_{2}\right)$, both purchased from BASF, for removing organic residues off the substrate. Afterward, they were dipped into a solution of $<5 \% \mathrm{HF}$ for $30 \mathrm{~s}$ in order to eliminate the native oxide layer. 
A variety of medium and high porosity PS monolayers were fabricated under galvanostatic conditions using anodization current densities of $5,11,16,27$ and $45 \mathrm{~mA} / \mathrm{cm}^{2}$, employing a custom-made vertical cell in which a Pt electrode worked as cathode and the silicon itself as anode. The etching times needed to create $1 \mu \mathrm{m}$ of PS were $125,70,50,35$, and $25 \mathrm{~s}$, respectively. Five series of samples were fabricated with these etching times multiplied by 1, 2, 5, 10 and 20 . An Agilent E3648A (Santa Clara, CA, USA) current generator was used, remotely controlled by a LabVIEW-based program.

Field emission scanning electron microscopy (FESEM) images of the PS monolayers were obtained using both a Hitachi S-4500 SEM (Chiyoda, Tokyo, Japan) and a Zeiss Ultra 55 (Oberkochen, Germany) microscope. The average thickness was statistically determined with Image J processing software. ${ }^{18}$

For the sample characterization, reflectance spectra measurements were performed using a Fourier-Transform Infrared Spectroscopy (FTIR) system (Bruker, Billerica, MA, USA) measuring in the NIR from 1100 to $2500 \mathrm{~nm}$. The spectrometer employed a liquid $\mathrm{N}_{2}$ cooled MTC326 detector. All spectra were recorded at $4 \mathrm{~cm}^{-1}$ resolution and averaged using 128 scans. Attenuated Total Reflection (ATR) FTIR spectra were also obtained with the former equipment in the range between 550 and $5000 \mathrm{~cm}^{-1}$, recorded at $6 \mathrm{~cm}^{-1}$ resolution and averaged using 1024 scans.

During the experiments, a type $\mathrm{K}$ thermocouple worked in the range from 323 to $453 \mathrm{~K}$. A Eurotherm 2216e (Worthing, UK) PID controller was used to increase the temperature $10 \mathrm{~K}$ every 5 minutes, avoiding overshooting.

All theoretical calculations as well as spectral fittings were carried out on MATLAB (R2016b, Mathworks, Natick, MA, USA). The effective medium approximation of Looyenga ${ }^{19}$ and Bruggeman ${ }^{20}$ were used to calculate the RI of the high and low porosity of the PS samples, respectively.

\section{Results and Discussion}

Five series of samples with a porosity between 50 and $75 \%$ were fabricated, having $1030 \pm 30 \mathrm{~nm}, 1780 \pm 90 \mathrm{~nm}, 4860 \pm 90 \mathrm{~nm}, 9700$ $\pm 800 \mathrm{~nm}$ and $16900 \pm 1000 \mathrm{~nm}$ of average thickness each and a pore diameter around $10 \mathrm{~nm}$.

During the experiments, the reflectance spectrum of the PS layers displayed a shift toward longer wavelengths when heat was applied (Figure 1a), as a result of the TOE. Besides, an intensity decay was also observed, which is related to the reflectance decay of silicon with temperature. The recorded data were used to calculate the RI at each temperature by means of spectral fitting of a mathematical model based on the Transfer Matrix Method (TMM). ${ }^{21}$ The obtained curves showed a linear relation, whose slope determined the TOC (Figure 1b), except for the samples of lower thickness (1 and $2 \mu \mathrm{m}$ series), which experienced a partial oxidation during the experiments, as will be discussed in a separate section.

Thermo-optic effect on PS in the NIR.-The thermal sensitivity at a given wavelength $\lambda$ is defined by: ${ }^{11}$

$$
\frac{d \lambda}{d T}=\lambda\left(\frac{1}{n} \frac{d n}{d T}+\frac{1}{h} \frac{d h}{d T}\right)
$$

where $n$ and $h$ are the RI and thickness of the monolayer, respectively. Both are temperature dependent due to the TOE and the thermal expansion effect (TEE). The latter is one order of magnitude lower than the former, ${ }^{9}$ which is similar to the error in our measurements, and for that reason we have disregarded the TEE in our approximation.

Experimental sensitivity was determined by measuring the spectral shift over time as the temperature increased. A simulation program based on the TMM was used to compare the experimental response of the PS monolayers with the theoretical one. In our program, the wavelength and temperature derivatives of silicon given by Li et al. ${ }^{22}$ were utilized to calculate the theoretical TOC of the PS samples.

In Figure 2, a comparison of the theoretical and experimental sensitivities of the PS monolayers at $1500 \mathrm{~nm}$ is depicted. A decrease of
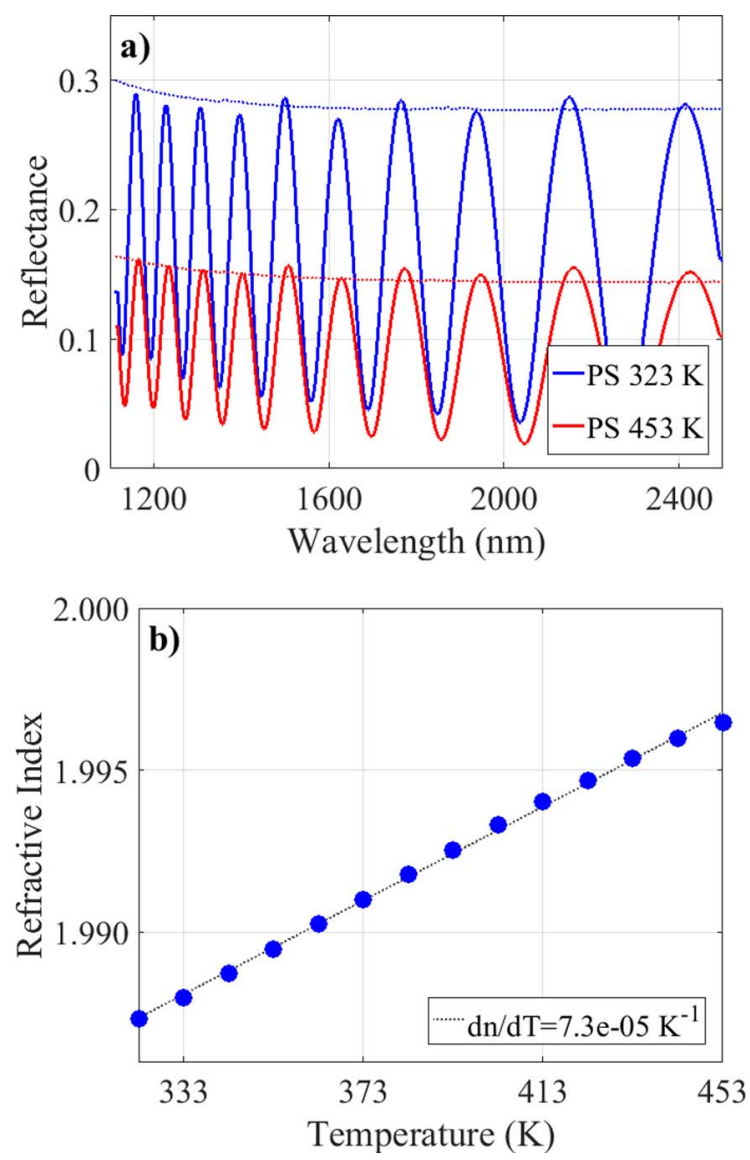

Figure 1. (a) Reflectance spectra of a silicon sample (dotted line) and a PS monolayer of $5 \mu \mathrm{m}$ and $55 \%$ of porosity (solid line) at room temperature (blue) and at $453 \mathrm{~K}$ (red). (b) RI of that PS monolayer at $1500 \mathrm{~nm}$ for each temperature.

the thermal sensitivity with increasing porosity was observed as well as a linear increase with wavelength. The experimental sensitivity values obtained were close to the theoretical calculations, but typically below them.

A maximum experimental sensitivity of $91 \pm 3 \mathrm{pm} /{ }^{\circ} \mathrm{C}$ was achieved during the experiments with one of the PS monolayers (56\% of porosity and $5 \mu \mathrm{m}$ at $2500 \mathrm{~nm}$ ). The sensitivity values at $1500 \mathrm{~nm}$ are higher than the ones achieved with a typical fiber-based sensor, ${ }^{23,24}$ but lower than that for an integrated photonic temperature sensor, ${ }^{11,25}$ as depicted in Table I. However, the lower cost and easier fabrication processes of PS temperature sensors are a great asset against the integrated photonic ones. Even though the sensitivity is, in theory, independent of the thickness of the layer, layers with lower thicknesses showed a smaller sensitivity during the experiments. As it will be discussed in the next section, this phenomenon can be explained by the partial oxidation of the samples.

Mathematical fitting of the reflectance spectra was carried out in order to extract the RI value of every PS sample at each temperature.

Table I. Thermal sensitivity comparison of our PS sensors with fiber-based (FB) temperature sensors and integrated photonics (IP) temperature sensors working at $1500 \mathrm{~nm}$.

\begin{tabular}{ccc} 
Type of structure & Sensitivity & Reference \\
\hline Fiber Bragg Grating (FB) & $10 \mathrm{pm} /{ }^{\circ} \mathrm{C}$ & Hirayama et al..$^{23}$ \\
Micro-Fiber Bragg Grating (FB) & $20 \mathrm{pm} /{ }^{\circ} \mathrm{C}$ & Kou et al. ${ }^{24}$ \\
Mach-Zehnder interferometer (IP) & $70 \mathrm{pm} /{ }^{\circ} \mathrm{C}$ & Lu et al..$^{25}$ \\
Silicon Fabry-Pérot cavity (IP) & $85 \mathrm{pm} /{ }^{\circ} \mathrm{C}$ & Liu et al. ${ }^{11}$ \\
Porous silicon films & $\mathrm{Up} \mathrm{to} 60 \mathrm{pm} /{ }^{\circ} \mathrm{C}$ & Our work
\end{tabular}



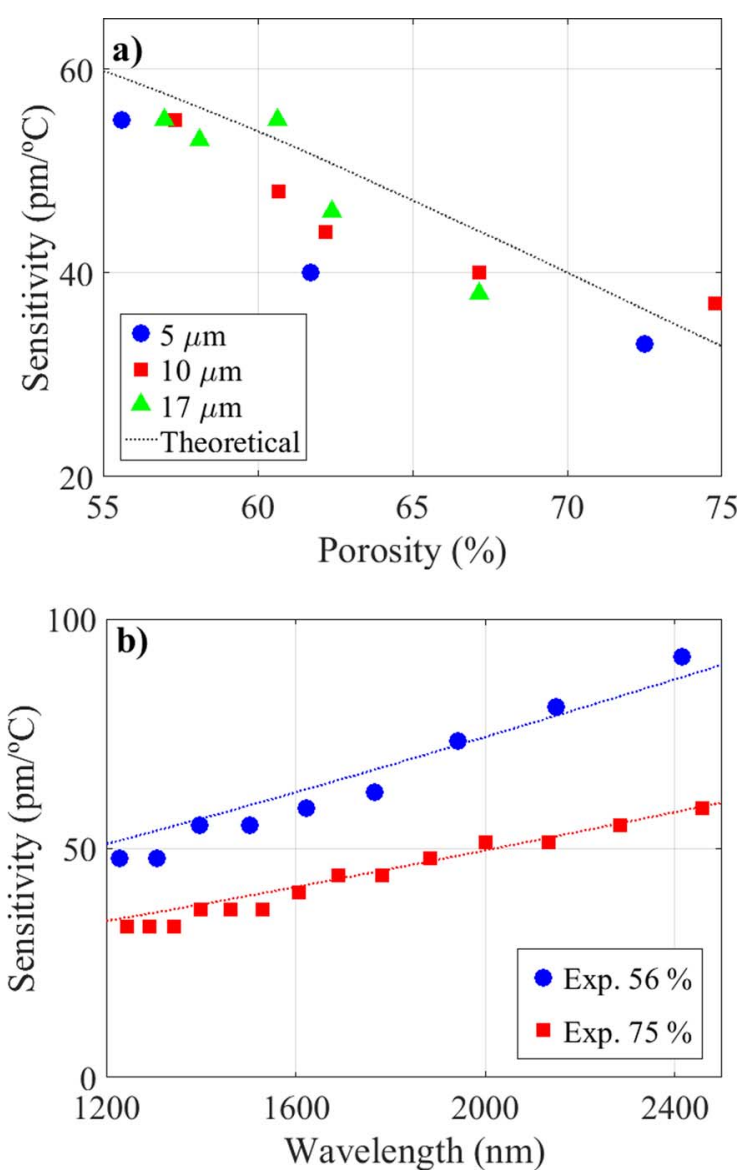

Figure 2. (a) Thermal sensitivity of the PS monolayers at $1500 \mathrm{~nm}$. (b) Maximum and minimum thermal sensitivities achieved in the NIR with the samples of $56 \%$ of porosity and $5 \mu \mathrm{m}$ (blue) and $75 \%$ of porosity and $10 \mu \mathrm{m}$ (red), respectively. Experimental sensitivities are represented as squares $\left( \pm 3 \mathrm{pm} /{ }^{\circ} \mathrm{C}\right)$ whereas the theoretical sensitivities are represented as dotted lines.

The TOC was calculated as a linear regression analysis of this scatter. The results are illustrated in Figure 3.

The experimental values were again slightly smaller than the theoretical calculations. We found that the TOC of the PS monolayers was between 3.2 and $7.9 \cdot 10^{-5} \mathrm{~K}^{-1}$ for the range of porosities we studied, which is consistent with the estimations of Moretti et al. ${ }^{9}$ The TOC evidenced an inverse relation with porosity, matching the results reported by López et al. ${ }^{26}$ Contrary to the tendency observed with the thermal sensitivity, however, this coefficient slightly decays with wavelength.

Modelling the oxidation process at low temperatures .- During the experiments, the samples with the lowest thicknesses displayed smaller spectral shifts than expected by the theoretical calculations and, in some cases, even backshifts toward shorter wavelengths. This behavior implied a reduction on the RI as the temperature increased (see Figure 4a). However, it could not be ascribed to the TOE, since the variation of the optical properties were permanent, as illustrated in Figure $4 b$.

Our results evidenced a physical change of the PS, which became more relevant as the thickness of the layer lowered, whereas its effect could be neglected when it was greater than $\sim 5 \mu \mathrm{m}$. This effect might be caused by the oxidation of silicon, whose outcome is a reduction on the RI, even though thermal oxidation usually requires higher temperatures. In consequence, we believe this is an initial phase of the oxidation process, in a dry air environment, at normal pressure and temperatures below $500 \mathrm{~K}$.

In order to characterize this effect, a spectral fitting program based on the TMM was developed to estimate the oxidation degree of each
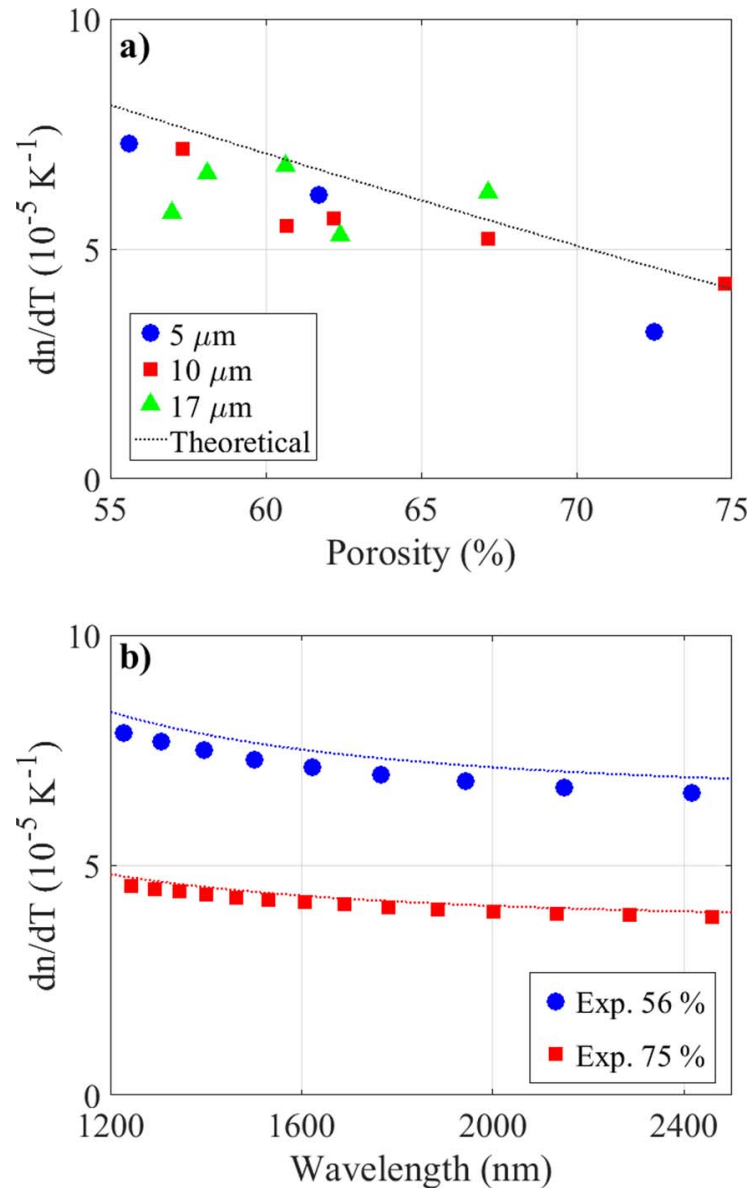

Figure 3. (a) TOC of the PS monolayers at $1500 \mathrm{~nm}$. (b) Maximum and minimum TOC achieved in the NIR with the samples of $55 \%$ of porosity and $5 \mu \mathrm{m}$ (blue) and $75 \%$ of porosity and $10 \mu \mathrm{m}$ (red), respectively. Experimental values are represented with markers whereas the theoretical sensitivities are represented as dotted lines.

sample from the reflectance measurements. At first, we modelled the pore as a cylinder with a homogeneous layer of oxide over the walls (see Figure 5a), using the pore model developed by Suárez et al. ${ }^{27}$ However, this model was not appropriate, since the porosity has a gradient with depth due to changes in the electrolyte during the electrochemical etching ${ }^{28}$ (Figure $5 \mathrm{~b}$ ).

A non-homogeneous PS layer was taken into account in the TMMbased program by splitting the monolayer on $\mathrm{N}$ layers, with $\mathrm{N}$ being high enough to satisfy:

$$
D_{k}^{-1} D_{k+1}=I,
$$

where $I$ is the identity matrix and $D$ is the matrix that describes the variation of the electromagnetic wave in the interface $k$ between two media. That assumption simplifies the definition of the transfer matrix as seen in the next equation:

$$
M=D_{0}^{-1} D_{1} \prod_{k=1}^{N} P_{k} D_{N}^{-1} D_{s} .
$$

The subscripts $O$ and $s$ refer to the initial medium and the substrate, respectively, and $P$ defines the propagation of the electromagnetic wave in the medium $k$. The multiplication matrix entails the summation of the exponents:

$$
\prod_{k=1}^{N} P_{k}=\left(\begin{array}{cc}
\exp \left(2 \pi \sum_{k=1}^{N}\left(h n_{k} / N\right) / \lambda\right) & 0 \\
0 & \exp \left(2 \pi \sum_{k=1}^{N}\left(h n_{k} / N\right) / \lambda\right)
\end{array}\right),
$$



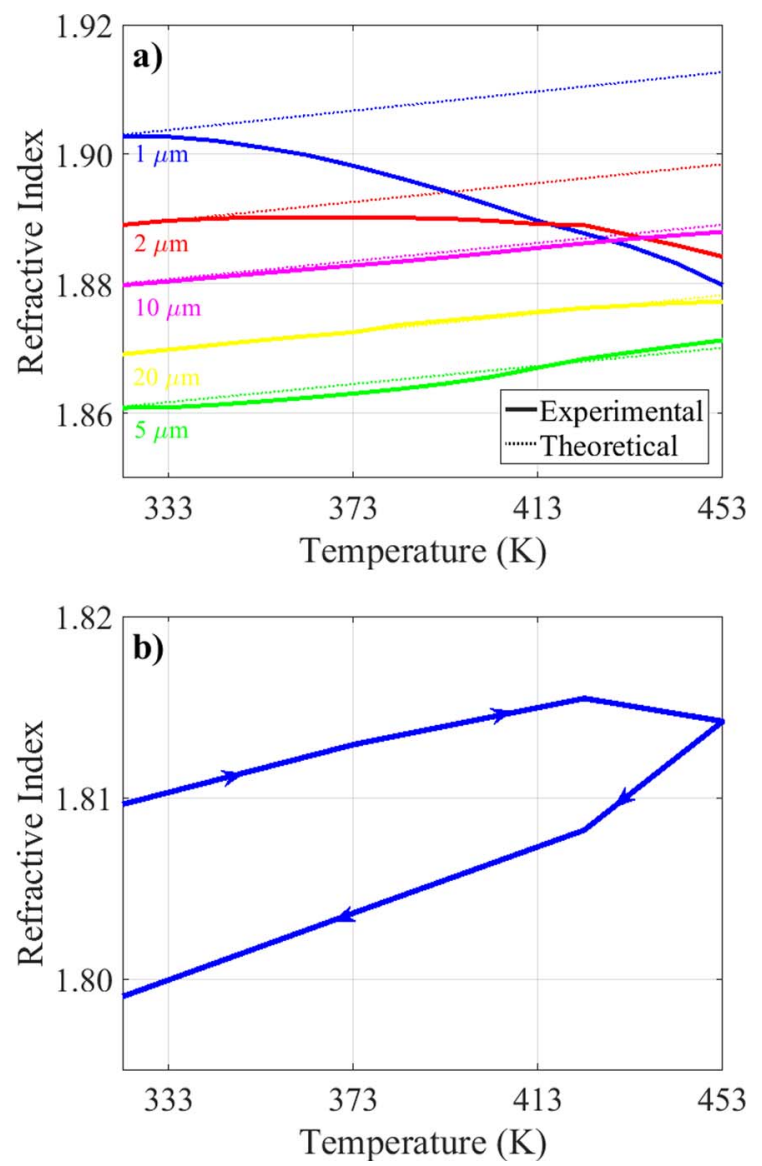

Figure 4. (a) RI variation with temperature of PS with similar porosity $(\sim 57 \%)$ and different thickness. Theoretical values are displayed as dotted lines whereas the experimental results are represented as solid lines. (b) RI evolution of a PS monolayer with $62 \%$ of porosity and $2 \mu \mathrm{m}$ in a cycle of heating and cooling.

which results in the numerical mean of the refractive index profile. Taking the previous equations into consideration, the reflectance spectrum of a non-homogeneous layer can then be expressed as:

$R=\frac{\left(1-n_{1} n_{s} / n_{0} n_{h}\right)^{2} \cos ^{2}\left(2 \pi h n_{\text {avg }} / \lambda\right)+\left(n_{s} / n_{h}-n_{1} / n_{0}\right)^{2} \sin ^{2}\left(2 \pi h n_{\text {avg }} / \lambda\right)}{\left(1+n_{1} n_{s} / n_{0} n_{h}\right)^{2} \cos ^{2}\left(2 \pi h n_{\text {avg }} / \lambda\right)+\left(n_{s} / n_{h}+n_{1} / n_{0}\right)^{2} \sin ^{2}\left(2 \pi h n_{\text {avg }} / \lambda\right)}$, a)

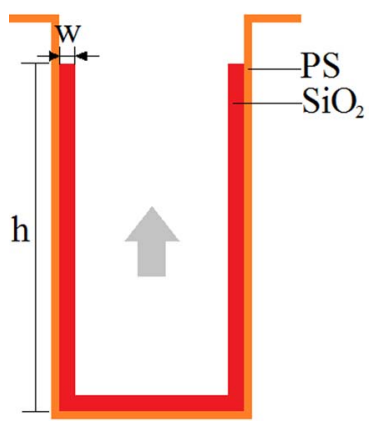

b)

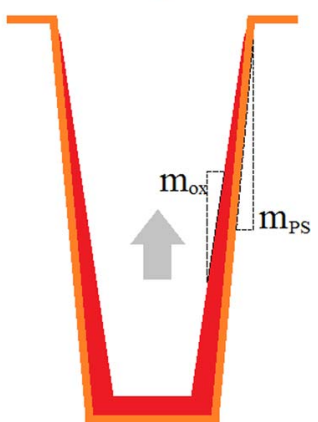

Figure 5. (a) Homogeneous pore model with a layer of silicon dioxide of width $w$, reaching a height $h$ of the total thickness. (b) Non-homogeneous model where the porosity has a gradient with depth, defined by the slop $m_{P S}$ and the silicon dioxide has a different gradient $m_{o x}$.
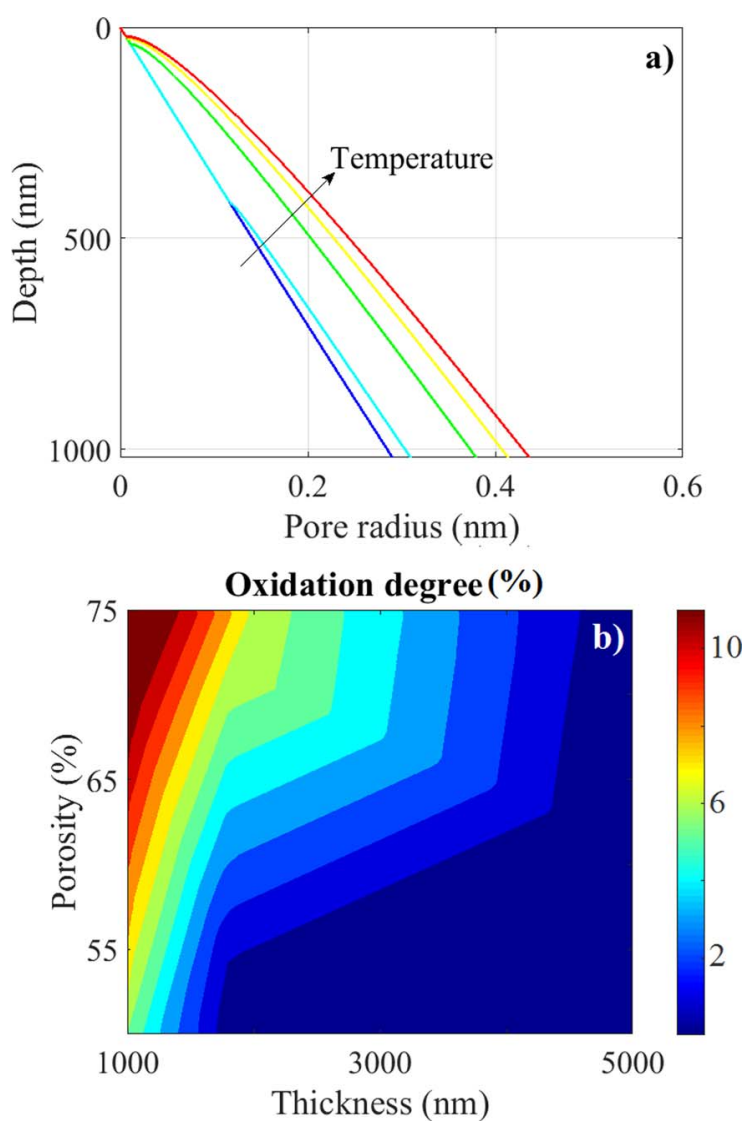

Figure 6. (a) Pore section profile of a monolayer with $60 \%$ of porosity and $1 \mu \mathrm{m}$. The pore radius is $0.3 \mathrm{~nm}$ lower at the pore tip with respect to the initial value. As the temperature increased, the width of the silicon oxide covering the pore walls grew bigger. (b) Oxidation degree of a PS monolayer for a temperature of $453 \mathrm{~K}$, depending on the porosity and the thickness, calculated as the percentage of silicon dioxide divided by the percentage of silicon. The oxidation degree of the samples with 10 and $17 \mu \mathrm{m}$ was the same as for those with $5 \mu \mathrm{m}$.

where $n_{\text {avg }}$ is the average refractive index of the monolayer. We used a linear variation approach of the RI profile. Thus, in order to estimate the degree of oxidation, we first performed a fitting of the reflectance spectrum at room temperature to determine the porosity at the surface of the structure and its variation slope. Our results indicated that the pore size slightly decreased with depth (see Figure 6a).

Following this first fitting, a second one was performed in order to characterize the RI variations with temperature, aside from the TOE. The results showed that the oxide grew wider with temperature. Besides, the silicon dioxide developed from the tip of the pores to the top, remaining thicker at the bottom (Figure $6 a$ ). In Figure $6 \mathrm{~b}$, the average percentage of oxidized PS at $453 \mathrm{~K}$ obtained for all the fabricated monolayers is presented.

The spectral fittings displayed a greater oxidation of the PS monolayers with lower thicknesses and higher porosities, which is consistent with the results obtained during the experiments. Moreover, the degree of oxidation of the samples with bigger thicknesses, although beneath $1 \%$, could explain the difference between the experimental values of the TOC and the thermal sensitivity with respect to the theoretical calculations.

We also performed several ATR-FTIR measurements on the samples used for the experiments in order to confirm this hypothesis. The monolayers with bigger thicknesses presented absorption peaks of $\mathrm{SiH}_{\mathrm{x}}(\mathrm{x}=3,2,1)$ stretching modes at 2142,2108 and $2087 \mathrm{~cm}^{-1}$, $\mathrm{SiH}_{2}$ scissor mode at $916 \mathrm{~cm}^{-1}$ and various $\mathrm{SiH}_{\mathrm{x}}$ deformation modes overlapping at 667 and $628 \mathrm{~cm}^{-1}$ (see Fig. 7). This spectrum profile is the typical of PS. ${ }^{16}$ However, the samples with lower thicknesses 

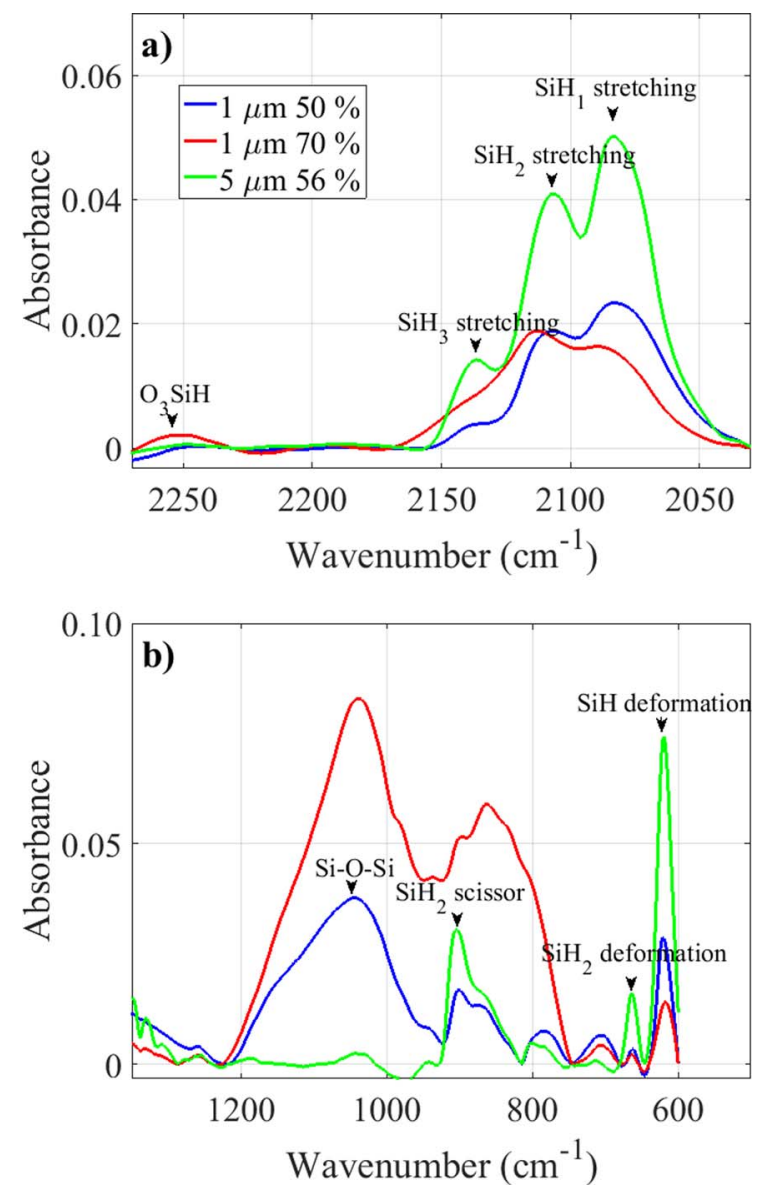

Figure 7. ATR-FTIR absorbance spectra of three PS monolayers after heating at $453 \mathrm{~K}$. (a) $\mathrm{SiH}_{\mathrm{x}}(\mathrm{x}=3,2,1)$ stretching modes at 2142,2108 and $2087 \mathrm{~cm}^{-1}$ and $\mathrm{Si}-\mathrm{H}$ stretching of $\mathrm{O}_{3} \mathrm{SiH}$ at $2256 \mathrm{~cm}^{-1}$. (b) $\mathrm{SiH}_{2}$ scissor bending mode at $916 \mathrm{~cm}^{-1}, \mathrm{SiH}_{2}$ wagging and $\mathrm{SiH}$ bending at 667 and $628 \mathrm{~cm}^{-1}$ and $\mathrm{Si}-\mathrm{O}-\mathrm{Si}$ antisymmetric stretching mode at $1050 \mathrm{~cm}^{-1}$.

displayed a different profile. The $\mathrm{SiH}_{\mathrm{x}}$ stretching (Figure 7a) and deformation (Figure $7 \mathrm{~b}$ ) modes suffered an intensity decrease. Besides, a direct proportion between the porosity and the decrease was observed. Around $1050 \mathrm{~cm}^{-1}$ a broadband mode started to develop, attributed to the $\mathrm{Si}-\mathrm{O}-\mathrm{Si}$ antisymmetric stretching. The $\mathrm{SiH}_{2}$ scissor mode also reduced its intensity and was overlapped by another broad band between 900 and $700 \mathrm{~cm}^{-1}$, which includes Si-O-Si symmetric stretching and $\mathrm{O}-\mathrm{Si}-\mathrm{H}$ deformation modes, among others. In the case of the sample with the highest porosity, the absorbance spectra showed the development of $\mathrm{Si}-\mathrm{H}$ stretching mode of $\mathrm{O}_{3} \mathrm{SiH}$ at $2256 \mathrm{~cm}^{-1}$ as well as a shift of the $\mathrm{SiH}_{\mathrm{x}}$ stretching modes toward higher frequencies. All these changes were consistent with the absorbance spectrum of partially oxidized PS. ${ }^{14}$ Particularly, the $\mathrm{SiH}_{\mathrm{x}}$ stretching modes should become a broad band centered at $2160 \mathrm{~cm}^{-1}$. In our case, those three peaks only shifted $10 \mathrm{~nm}$ which, together with the missing $\mathrm{Si}-\mathrm{H}$ stretching mode of $\mathrm{O}_{2} \mathrm{SiH}_{2}$ at $2200 \mathrm{~cm}^{-1}$, indicated that the samples were on an initial phase of the oxidation process.

\section{Conclusions}

The porosity of a porous silicon structure is a key parameter for tuning the thermal properties of this material. As reported in this work, the TOC can be changed between 3.2 and $7.9 \cdot 10^{-5} \mathrm{~K}^{-1}$ in a range of porosities from 50 to $75 \%$. We performed the characterization in the infrared region, where a wide variety of applications work, and obtained values lower than in the visible range. According to our results, the thickness of the layer is also of great importance. Lower thicknesses favor the oxidation of the structures and, thus, present smaller TOC and thermal sensitivities.

We report evidence of oxidation at low temperatures $(<500 \mathrm{~K})$. The initial phase of this process consists on the growth of a thin silicon dioxide layer over the pore walls, causing a backshift of the reflectance spectrum and a slight change of the absorbance modes in the ATR-FTIR analysis. A mathematical model was developed to estimate the oxidation degree from reflectance spectra and the results were consistent with the experimental measurements. This approximation can be useful when other methods for measuring the oxidation of a sample are not available, i.e. weighting the sample or performing ATR-FTIR measurements. One remaining question is how the resistivity influences the thermo-optical effect of porous silicon, since our experiments were performed using only heavily doped p-type silicon wafers.

\section{Acknowledgments}

The authors acknowledge the funding from the Spanish government through the project TEC2015-63838-C3-1-ROPTONANOSENS.

\section{ORCID}

David Martín-Sánchez (D) https://orcid.org/0000-0003-0131-5226

\section{References}

1. J. Carstensen and G. Hasse, Mater. Sci. Eng. R., 39(4), 93 (2002)

2. A. Loni, in Handbook of porous silicon, L.T. Canham, Springer International Publishing, Switzerland, 2014.

3. O. Bisi, S. Ossicini, and L. Pavesi, Surf. Sci. Rep., 38(1-3), 6 (2000).

4. V. Torres-Costa and R. J. Martín-Palma, J. Mater. Sci., 45, 2823 (2010)

5. W. Li, Z. Liu, F. Fontana, Y. Ding, D. Liu, J. T. Hirvonen, and H. A. Santos, $A d v$ Mater., 30, 1703740 (2018).

6. R. Yi, F. Dai, M. L. Gordin, S. Chen, and D. Wang, Adv. Energy Mater., 3, 295 (2013)

7. N. Liu, K. Huo, M. T. McDowell, J. Zhao, and Y. Cui, Sci. Rep., 3, 1919 (2013).

8. M. Ashuri, Q. He, and L. L. Shaw, Nanoscale, 8, 74 (2016).

9. L. Moretti, L. Stefano, A. M. Rossi, and I. Rendina, Appl. Phys. Lett., 86, 061107 (2005).

10. G. Cocorullo, F. G. Corte, I. Rendina, and P. M. Sarro, Sens. Actuators A Phys., 71, 19 (1998)

11. G. Liu, M. Han, and W. Hou, Opt. Express, 23(6), 7237 (2015).

12. J. Sik, J. Hora, and Humlicek, J. Appl. Phys., 84(11), 6291 (1998).

13. A. E. Pap, K. Kordás, T. F. George, and S. Leppävuori, J. Phys. Chem. B, 108(34), 12744 (2004).

14. Y. H. Ogata, T. Tsuboi, T. Sakka, and S. Naito, J. Porous Mat., 7, 63 (2000).

15. T. Unagami, Jpn. J. Appl. Phys., 19(2), 231 (1980).

16. D. B. Mawhinney, J. A. Glass, and J. T. Yates, J. Phys. Chem. B, 101, 1202 (1997).

17. C. Baker and J. L. Gole, JSM Nanotechnol. Nanomed., 2(1), 1021 (2014).

18. W. S. J. Rasband, U. S. National Institutes of Health, Bethesda, Maryland, USA, 1997.

19. H. Looyenga, Physica, 31(3), 401 (1965).

20. D. A. G. Bruggeman, Ann. Phys., 416(7), 636 (1935).

21. R. B. Balili, Int. J. Mod. Phys., 17, 159 (2012).

22. H. H. Li, J. Phys. Chem. Ref. Data, 9(3), 561 (1980).

23. N. Hirayama and Y. Sano, ISA T., 39, $169(2000)$

24. J. Kou, S. Qiu, F. Xu, and Y. Lu, Opt. Express, 19, 18452 (2011)

25. P. Lu, L. Men, K. Sooley, and Q. Chen, Appl. Phys. Lett., 94, 131110 (2009).

26. L. P. López, A. I. Mabilangan, M. A. B. Faustino, N. G. E. Saplagio, A. A. Salvador, and A. S. Somintac, Int. J. Electrochem. Sci., 9, 8079 (2014).

27. I. Suárez, V. Chirvony, D. Hill, and J. Martínez-Pastor, Photonic Nanostructures: Fundam. Appl., 10(3), 304 (2012)

28. S. Billat, M. Thönissen, R. Arens-Fischer, M. G. Berger, M. Krüger, and H. Lüth, Thin Solid Films, 297, 22 (1997). 